\begin{tabular}{|c|l|}
\hline Title & Effects of 8-hydroxy-GTP and 2-hydroxy-A TP on in vitro transcription. \\
\hline Author(s) & Kamiya, Hiroyuki; Suzuki, A kihiro; Kawai, Kazuaki; Kasai, Hiroshi; Harashima, Hidey oshi \\
\hline Citation & $\begin{array}{l}\text { Free Radical Biology and Medicine, 43(5), 837-843 } \\
\text { https://loi.org/10.1016/.freeradbiomed.2007.05.034 }\end{array}$ \\
\hline Issue Date & 2007-09-01 \\
\hline Doc URL & http://hdl.handle.net/2115/27982 \\
\hline Type & article (author version) \\
\hline File Information & FRBM43-5.pdf \\
\hline
\end{tabular}

Instructions for use 


\title{
Effects of 8-hydroxy-GTP and 2-hydroxy-ATP on in vitro transcription
}

\author{
Hiroyuki Kamiya ${ }^{\mathrm{a}, *}$, Akihiro Suzukia , Kazuaki Kawai ${ }^{\mathrm{b}}$, Hiroshi Kasai', \\ and Hideyoshi Harashima ${ }^{a}$
}

${ }^{a}$ Faculty of Pharmaceutical Sciences, Hokkaido University, Kita-12,

Nishi-6, Kita-ku, Sapporo 060-0812, Japan

${ }^{\mathrm{b}}$ Institute of Industrial Ecological Sciences, University of Occupational and Environmental Health, 1-1 Iseigaoka, Yahatanishi-ku, Kitakyushu 807-8555, Japan

*Corresponding author: Tel +81-11-706-3733, Fax +81-11-706-4879, E-mail hirokam@pharm.hokudai.ac.jp

This work was supported in part by Grants-in-Aid from the Japan Society for the Promotion of Science.

Running title: Oxidized ribonucleotides 
Title: Effects of 8-hydroxy-GTP and 2-hydroxy-ATP on in vitro transcription

\begin{abstract}
Oxidation of RNA precursors may disturb genetic information. In this study, the effects of oxidized RNA precursors on in vitro transcription were examined. Two oxidized ribonucleoside triphosphates, 8-hydroxyguanosine 5'-triphosphate (8-OH-GTP) and 2-hydroxyadenosine 5'-triphosphate (2-OH-ATP), were added to in vitro transcription reactions. The addition of 8-OH-GTP and 2-OH-ATP reduced the amount of RNA synthesized in vitro. Moreover, to examine qualitative alteration of the mRNA, it was converted to cDNA by reverse transcriptase, and the cDNA was then amplified by PCR. The PCR product was subsequently cloned into plasmid DNA, and the DNA sequence was analyzed for each bacterial colony. The two oxidized ribonucleotides induced mutations in cDNA, suggesting the disturbance of genetic information during transcription and/or reverse transcription. 8-OH-GTP induced $\mathrm{T} \rightarrow \mathrm{G}$ plus $\mathrm{T} \rightarrow \mathrm{C}$ mutations, and 2-OH-ATP caused $\mathrm{T} \rightarrow \mathrm{C}$ mutations. These results indicate that the formation of these oxidized RNA precursors in cells affects transcription quantitatively and qualitatively.
\end{abstract}

Keywords: ribonucleotide; 8-hydroxy-GTP; 2-hydroxy-ATP; transcription 


\section{Introduction}

Oxidation of nucleic acids and their related compounds occurs endogenously by normal oxygen metabolism, and is also induced by many environmental mutagens and carcinogens. For these reasons, DNA oxidation seems to be a very important source of mutations as well as one of the causative factors of carcinogenesis, neurodegeneration, and aging [1-3]. In addition, the oxidation of DNA precursors in the nucleotide pool by ROS is another significant source in the mutation process. The importance of oxidized DNA precursors is highlighted by the presence of Escherichia coli MutT and its functional homologues, and by the phenotypes of mutant organisms lacking the MutT-type enzymes [ref. 4 and references therein]. The direct introduction of oxidized DNA precursors into living cells induces mutations in chromosomal genes, providing another line of evidence that they are mutagens when produced in cells $[5,6]$.

RNA and its precursors are also oxidized by ROS. The oxidized RNA was reportedly implicated in several neurodegenerative diseases [7-10]. Because the concentrations of RNA precursors are $0.1-3 \mathrm{mM}$ in living cells and much higher than those of DNA precursors, oxidized RNA precursors may be more abundant than oxidized DNA precursors $[11,12]$. Previously, a relationship between the oxidation of an RNA precursor and 
transcriptional errors was suggested. In the mut $T$ strain, a protein containing an altered sequence was produced with higher frequency than that expected from the mutation frequency at the DNA level [13]. The authors of that paper concluded that an oxidized form of GTP, 8-OH-GTP (also known as 8-oxo-7,8-dihydroguanosine 5'-triphosphate), was misincorporated opposite A by RNA pol and induced translational errors, because the MutT protein hydrolyzes $8-\mathrm{OH}-\mathrm{GTP}$ to the monophosphate [13]. The findings that some MutT-type enzymes including human MTH1 protein catalyze the hydrolysis of oxidized RNA precursors suggest the importance of the hydrolytic elimination of oxidized ribonucleotides [ref. 4 and references therein]. Moreover, the yeast S-II null mutant exhibits sensitivity to oxidants and is defective in maintaining transcriptional fidelity [14]. This low transcriptional fidelity is relieved by treatment with an antioxidant, suggesting that the yeast S-II protein maintains transcriptional fidelity by enhancing the cleavage of misincorporated, oxidized ribonucleotides from nascent mRNA [14]. Thus, the formation of oxidized RNA precursors in the nucleotide pool causes disturbances in the genetic information, affecting transcription and occasionally leading to cell death. The oxidized RNAs reportedly related to several neurodegenerative diseases [7-10] may be formed by incorporation of the oxidized RNA precursors. 
In this study, we focused on the effects of oxidized ribonucleotides

during transcription. 8-OH-GTP and oxidized ATP, 2-OH-ATP, were chosen as representative oxidized ribonucleoside 5'-triphosphates, because they are produced as the major products in in vitro oxidation reactions of GTP and ATP, respectively [15]. We found that the presence of 8-OH-GTP and 2-OH-ATP reduced the amounts of full-length mRNA in transcription. In addition, the two oxidized RNA precursors induced mispair formation. These results suggest the biological effects of the two damaged ribonucleotides.

\section{Materials and Methods}

\section{Materials}

T7 RNA polymerase and ribonuclease inhibitor were purchased from Takara (Kusatsu, Japan). The pTENHES plasmid [16] was amplified in the E. coli strain DH5 $\alpha$ and was purified with a Qiagen (Hilden, Germany) EndoFree Plasmid Mega kit. 8-OH-GTP and 2-OH-ATP were prepared by oxidation of GTP and ATP, respectively, as described previously [15]. The purities of $8-\mathrm{OH}-\mathrm{GTP}$ and 2-OH-ATP were both estimated to be more than $99 \%$ by analysis with HPLC (data not shown). 
The unmodified ribonucleoside 5'-triphosphates, Cy5-UTP, and Cy3-UTP were from GE Healthcare Bio-Sciences (Piscataway, NJ). Purified oligonucleotides were from Sigma Genosys Japan (Ishikari, Japan).

In vitro transcription

The pTENHES plasmid was digested with ScaI to produce a 3495-bp DNA fragment containing the T7 promoter and $2 / 3$ of the $\mathrm{N}$-terminal region of the Hyg-EGFP gene (fusion gene of the hygromycin resistance gene and enhanced green fluorescence protein gene). In vitro transcription was carried out essentially according to the supplier's instructions, by incubating the ScaI fragment (35 fmol) and T7 RNA pol $(15 \mathrm{U})$ at $37^{\circ} \mathrm{C}$ for $2 \mathrm{hr}$, in a reaction mixture $(6.67 \mu \mathrm{l})$ containing $40 \mathrm{mM}$ Tris-HCl, $\mathrm{pH} 8.0,8 \mathrm{mM} \mathrm{MgCl}_{2}, 2.0 \mathrm{mM}$ spermidine, $5.0 \mathrm{mM}$ dithiothreitol, $1.0 \mathrm{mM}$ ribonuclease inhibitor, and the four ribonucleoside 5'-triphosphates ( 0.5 or $2 \mathrm{mM}$ each), with or without an oxidized ribonucleotide (various concentrations) and a fluorescently labeled ribonucleotide (Cy3- or Cy5-UTP, $0.4 \mathrm{mM}$ ). Reactions were terminated by the addition of $13.5 \mu 1$ of blocking buffer (29.6 mM MOPS, $\mathrm{pH} 7.0,7.4 \mathrm{mM}$ sodium acetate, 1.5 mM EDTA, $18.5 \%$ formaldehyde, $74.1 \%$ formamide) and subsequent incubation at $65^{\circ} \mathrm{C}$ for $15 \mathrm{~min}$. The reaction mixtures were analyzed by $18 \%$ formaldehyde-1\% agarose gel electrophoresis. When the transcription 
reaction was carried out without a fluorescently labeled ribonucleotide, the gel was stained with SYBR-Gold (Invitrogen, Carlsbad, CA). Fluorograms were obtained with a Fujifilm FLA-2000 Fluorescent Image Analyzer (Fuji Photo Film, Tokyo, Japan). The relative mRNA amounts were calculated based on the intensity of the experimental band.

The mRNAs obtained by the in vitro transcription without a fluorescently labeled ribonucleotide were treated with DNase and purified with an RNeasy mini column (Qiagen). The purified mRNAs were used for the RT reactions and the 8-OH-Guo analyses described below.

$R T$-PCR and sequence analysis

First-strand cDNA synthesis was performed on the purified mRNA using a primer (Primer-AS, 5'-dAAAAAGCTTACTTCTACACAGCCATCGGT) and an RNA PCR Kit (AMV) (Takara) according to the manufacturer's instructions. Each of the mRNA transcripts was amplified by PCR, using the Primer-AS and another primer (Primer-S, 5'-dAAAACTAGTGGGgAATTGTGAGCGGATAA). The PCR product thus obtained was blunted by a Blunting high kit (Toyobo, Osaka, Japan).

The DNA was inserted into pBluescript II SK(+), which had been digested with EcoRV. The ligated DNA was transfected into the E. coli 
strain DH5 $\alpha$, and the white colonies on agar plates containing 5-bromo-4-chloro-3-indolyl- $\beta$-D-galactoside, isopropyl- $\beta$-D-thiogalactopyranoside and ampicillin were selected.

The nucleotide sequences were analyzed by sequencing using an ABI PRISM Big Dye Terminator Cycle Sequencing Kit and an ABI model 377 DNA sequencer (Applera, Norwalk, CT).

\section{8-OH-Guo determination by HPLC}

The mRNAs obtained in the presence of 8-OH-GTP were purified as described above, and then were treated with nuclease P1 and calf intestine alkaline phosphatase. The amount of $8-\mathrm{OH}-\mathrm{Guo}$ was determined by HPLC, essentially as described previously [17]. Namely, the nucleoside solution was filtered through an Ultrafree-Probind filter (Millipore, Bedford, MA). The filtrate was then injected into a HPLC column (Capcellpak C18 MG, 4.6 X 250 mm, $5 \mu \mathrm{m}$, Shiseido Fine Chemicals, Tokyo, Japan) equipped with UV (UV-8020, Tosoh, Tokyo, Japan) and electrochemical (ECD-300, Eicom, Kyoto, Japan) detectors. The mobile phase consisted of $8 \%$ methanol, $10 \mathrm{mM} \mathrm{NaH}{ }_{2} \mathrm{PO}_{4}$ and $50 \mathrm{mg} / \mathrm{l} \mathrm{EDTA} \cdot 2 \mathrm{Na}$ was used for elution at a flow rate of $1.0 \mathrm{ml} / \mathrm{min}$. The effluent was monitored by UV absorption at $260 \mathrm{~nm}$ for guanosine and by electrochemical response (applied voltage, $550 \mathrm{mV}$ ) for 8-OH-Guo. The 
8-OH-Guo level in the RNA sample was calculated from HPLC runs of standard samples of 8-OH-Guo and guanosine.

\section{Results}

Reduced mRNA production in the presence of oxidized ribonucleotides

We first examined the effects of 8-OH-GTP and 2-OH-ATP on the yield of mRNA produced in the in vitro transcription reactions. T7 RNA pol was used as a model RNA pol, due to its strong enzymatic activity (Fig. 1). The reaction conditions were compatible with those recommended by the company supplying the pol. In addition to the four unmodified NTPs (2 $\mathrm{mM}$ each), various concentrations of 8-OH-GTP or 2-OH-ATP were added into the reaction mixtures. The in vitro transcription reactions were conducted in the presence of a fluorescent UTP analog, and the mRNAs produced in the reactions were visualized with a fluorescent image analyzer. We quantified the band intensities with the analyzer and represented them as the amounts of mRNA. Cy3- or Cy5-UTP was added into the transcription reactions for the quantitation. The amounts of the full-length transcript $\quad\left(\begin{array}{ll}\sim 1.2 & \mathrm{~kb})\end{array}\right)$ were reduced in an 8-OH-GTP concentration-dependent manner (Table 1). In the presence of 1 and $2 \mathrm{mM}$ 
8-OH-GTP, the amounts of the mRNA were $55 \%$ and $16 \%$, respectively, of that in the absence of 8-OH-GTP. Likewise, 2-OH-ATP decreased the amount of the mRNAs produced in the in vitro transcription reaction (Table 1). One and $2 \mathrm{mM}$ 2-OH-ATP reduced the yield of the mRNA by $50 \%$ and $75 \%$, respectively. This reduction in the amounts of the mRNA, at least qualitatively, was not due to artifacts derived from competition between a fluorescent UTP analog and an oxidized ribonucleotide, because the full-length transcripts, obtained in the transcription reactions without a fluorescent UTP analog, were also decreased when the gel was stained with SYBR-Gold (data not shown). Similar decreases in the mRNA yield were observed when the unmodified and oxidized ribonucleotides were each added at a concentration of $0.5 \mathrm{mM}$ (Table 1$)$.

Detection of 8-OH-Guo in mRNA

We next examined whether an oxidized RNA precursor was actually incorporated into the transcript. Since the 8-OH-Gua nucleoside can be detected with high sensitivity by HPLC-ECD [18], we analyzed the mRNA obtained in the presence of $2 \mathrm{mM} 8-\mathrm{OH}-\mathrm{GTP}$ by this method. The purified mRNA was treated with nuclease P1 and calf intestine alkaline phosphatase, and the nucleosides were analyzed by HPLC-ECD. 
The average amount of 8-OH-Guo was $2.4(1.9$ and 2.8$)$ per $10^{4}$ Guo in the mRNA produced in the absence of 8-OH-GTP, where the values in the parentheses represent those obtained in a single experiment. This high level of $8-\mathrm{OH}-\mathrm{Gua}$ in the control mRNA might be derived from incorporation of 8-OH-GTP formed by spontaneous oxidation of GTP and/or direct oxidation of $\mathrm{G}$ residues in the mRNAs during incubations with enzymes. As expected, the 8-OH-Gua content was much higher in the mRNA transcribed with 8-OH-GTP. The average amount of 8-OH-Guo was 430 (400.9 and 458.9) per $10^{4}$ Guo in the mRNA, where the values in the parentheses represent those obtained in a single experiment. Thus, 8-OH-GTP was incorporated by T7 RNA pol with an efficiency of $4 \%$ as compared to that of GTP. A single molecule of the mRNA transcribed with 8-OH-GTP was calculated to contain nearly ten 8-OH-Guo nucleosides.

Mispair formation during transcription and reverse transcription

We next examined qualitative alteration of the mRNA. We analyzed the effects of $8-\mathrm{OH}-\mathrm{GTP}$ and 2-OH-ATP on the fidelities of transcription-reverse transcription. Reverse transcriptase could induce errors at the $8-\mathrm{OH}-\mathrm{Gua}$ and 2-OH-Ade positions and thus the mutations possibly indicate the sites where 8 -OH-GTP and 2-OH-ATP were incorporated. After DNase treatment, the transcripts obtained with the in 
vitro reactions in the presence of $8-\mathrm{OH}-\mathrm{GTP}$ or $2-\mathrm{OH}-\mathrm{ATP}$ were purified by a commercially available silica-gel membrane device. No product was detected after PCR when the mRNA fraction purified by this procedure was added as the 'template' (data not shown), excluding the possibility of DNA contamination. The transcripts were reverse transcribed with AMV RT, and their cDNAs were amplified by PCR. The PCR products thus obtained were inserted into the pBluescript II SK(+) plasmid, and the sequences of plasmids in isolated colonies were determined (Fig. 1).

We analyzed the sequences in 17,17 , and 26 colonies, obtained with the control (without an oxidized ribonucleotide), 8-OH-GTP, and 2-OH-ATP experiments, respectively. As shown in Table 2, among the 17 clones, 3 contained a mutation in the case of the control experiment. These mutations might have occurred during the RT reaction and/or amplification (PCR). 8-OH-GTP strongly induces mispair formation. Mutations were found in most of the clones analyzed, and several clones contained errors at two sites. In the case of 2-OH-ATP, the error frequency was slightly increased in comparison with the control experiment. Among the 26 clones, 10 contained a mutation. Thus, $8-\mathrm{OH}-\mathrm{GTP}$ and 2-OH-ATP affected the RNA pol fidelity and/or the 8-OH-Gua and 2-OH-Ade residues in RNA induced misincorporation by RT. 
All of the mutations detected in these experiments were substitutions. Here, we describe the sequence changes in the sense strand, which corresponds to the mRNA sequence (Table 2). In the case of 8-OH-GTP, $\mathrm{T} \rightarrow \mathrm{G}$ and $\mathrm{T} \rightarrow \mathrm{C}$ mutations were induced. The $\mathrm{T} \rightarrow \mathrm{G}$ mutations were found in $\sim 50 \%$ of the analyzed clones. These types of mutations are analogous to the A:T $\rightarrow \mathrm{C}: \mathrm{G}$ transversions induced by 8-OH-dGTP [5] (see Discussion). Nearly $35 \%$ of the analyzed clones contained $\mathrm{T} \rightarrow \mathrm{C}$ mutations. This type of mutation had not been expected, because the corresponding deoxyribonucleotide, 8-OH-dGTP, does not induce transition mutations. Thus, the induction of the $\mathrm{T} \rightarrow \mathrm{C}$ mutations might be specific for in vitro transcription in the presence of 8-OH-GTP and the subsequent reverse transcription. The $\mathrm{T} \rightarrow \mathrm{C}$ mutations occurred in the 2-OH-ATP experiment, but not in the control experiment (Table 2).

The distributions of the mutations induced by $8-\mathrm{OH}-\mathrm{GTP}$ and 2-OH-ATP are shown in Table 3 and Fig. S1. The mutations caused by 8-OH-GTP were uniformly located, and no major mutational hotspot was observed. In the case of 2-OH-ATP, the same two mutations were found at three sites. However, the conclusion that 2-OH-ATP induced the $\mathrm{T} \rightarrow \mathrm{C}$ mutations seems to be relevant, since $\mathrm{T} \rightarrow \mathrm{C}$ mutations were found at four sites. 


\section{Discussion}

The objective of this study was to examine the effects of oxidized ribonucleotides on the quantity as well as the quality of mRNA, because oxidized ribonucleotides have been suggested to be involved in transcriptional (and translational) errors and cell death $[13,14]$. We found that $8-\mathrm{OH}-\mathrm{GTP}$ and 2-OH-ATP reduced the production of the full-length mRNAs (Table 1). One possible explanation is that the incorporation of the oxidized ribonucleotides and/or the elongation from the 3'-terminal, incorporated oxidized ribonucleotide is inefficient, resulting in truncated mRNA production. In the presence of $2 \mathrm{mM} \mathrm{8-OH-GTP}$ and 2-OH-ATP, the yields of the full-length transcript were $~ 20 \%$ (Table 1). Even if 8-OH-GTP was incorporated only opposite $\mathrm{C}, \sim 250$ kinds of truncated products could be present. However, we did not detect the putative truncated products on the gel visualized by a fluorescent nucleotide (Table 1) or SYBR-Gold staining (data not shown). Although it is difficult to detect short RNA fragments by either the incorporated fluorescent nucleotide or staining with SYBR-Gold (because longer RNA fragments contain more fluorescent nucleotides or bind higher amounts of the dye), this explanation seems to be unsatisfactory for the $\sim 80 \%$ reduction in the 
yields. Alternatively, interaction of the oxidized ribonucleotides with T7 RNA pol might decrease the full-length transcript. Purine ribonucleotides, GTP and ATP, have high binding affinities for T7 RNA pol. The binding induces a conformational change of the pol during initiation [19]. The effects of GTP and ATP on the stabilities of DNA-RNA pol complexes and on RNA production are different [20]. Thus, it is possible that 8-OH-GTP and 2-OH-ATP bind to T7 RNA pol and cause it to undergo a conformational change. The induced conformational change might decrease the production of mRNA. As shown in Table 1, the effects of 8-OH-GTP and 2-OH-ATP on the reduction in the full-length products were slightly different when they were present in $0.50 \mathrm{mM}$ concentration. This may be due to differences in the degrees of the two inhibitory effects of 8-OH-GTP and 2-OH-ATP.

8-OH-GTP induced $\mathrm{T} \rightarrow \mathrm{G}$ mutations during transcription and reverse transcription (Table 2). The $\mathrm{T} \rightarrow \mathrm{G}$ mutations seemed to be caused by the incorporation of $8-\mathrm{OH}-\mathrm{GTP}$ opposite $\mathrm{A}$ in the template DNA by T7 RNA pol (Fig. 2A). When dCTP was incorporated opposite the incorporated 8-OH-Gua residue in RNA during the reverse transcription, the A base at the original position was converted to the $\mathrm{C}$ base (the $\mathrm{A} \rightarrow \mathrm{C}$ mutation in the antisense strand is equivalent to the $\mathrm{T} \rightarrow \mathrm{G}$ mutation in the sense strand). This result agrees with the finding that E. coli RNA pol 
incorporated 8-OH-GTP into poly(dA-dT), probably opposite A [13]. 8-OH-Gua:A and 8-OH-Gua:C pairs are formed in the DNA duplexes [21-24]. These mutations are equivalent to the $A: T \rightarrow C: G$ transversions induced by the corresponding oxidized DNA precursor, 8-OH-dGTP [5].

8-OH-GTP also induced $\mathrm{T} \rightarrow \mathrm{C}$ mutations (Table 1). This type of mutation is explained by the incorporation of $8-\mathrm{OH}-\mathrm{GTP}$ opposite A by T7 RNA pol and the subsequent insertion of dGTP opposite the incorporated 8-OH-Gua residue by AMV RT (Fig. 2B).

2-OH-ATP also caused $\mathrm{T} \rightarrow \mathrm{C}$ mutations (Table 1). As with 8-OH-GTP, this type of mutation is explained by the incorporation of 2-OH-ATP opposite A by T7 RNA pol and the subsequent insertion of dGTP opposite the incorporated 2-OH-Ade residue by AMV RT (Fig. 2C). 2-OH-Ade reportedly can pair with any of the four unmodified bases $[25,26]$. This result is in contrast to those reported by Bukowska and Kuśmierek, who found that deoxyribonucleotides other than dTTP were incorporated opposite 2-OH-Ade in RNA at only a low level, if any, by AMV RT [27]. We cannot account for this discrepancy at this moment. However, they used copolymers including 2-OH-Ade as templates. Misincorporation opposite 2-OH-Ade in RNA might occur in a sequence context-dependent manner. Although the mutation frequencies in the presence and absence of 2-OH-ATP differed by only two-fold (Table 2), 
the mutations found in the control (without 2-OH-ATP) experiment were expected to occur upon transcription-reverse transcription and/or during PCR. The error rate of T7 RNA pol is $3-5 \times 10^{-5}[28,29]$. On the other hand, the error rate of AMV RT on an RNA template is $\sim 1.5 \times 10^{-4}[30]$. In the control experiment, three mutations were found in the 17 colonies examined. Since we analyzed a region of 1133 bp per colony, the mutation frequency was calculated to be $1.6 \times 10^{-4}$. Judging from the reported error frequencies, the mutations found in the control experiment could occur in the reverse transcription, although no information on the fidelity of the DNA pol used in PCR is available. In the 2-OH-ATP experiment, the mutation frequency was calculated to be $3.4 \times 10^{-4}$. Thus, 2-OH-ATP seemed to enhance mutations in transcription-reverse transcription.

As described above, 8-OH-GTP and 2-OH-ATP might bind to T7 RNA pol and change its conformation. It is conceivable that the RNA pol with an altered conformation is error-prone and inserts unmodified ribonucleotides opposite incorrect bases during transcription. This is another possible mechanism for the mutations detected in the DNA after reverse transcription and PCR.

Although we used T7 phage RNA pol as a model RNA pol in this study, the incorporation of $8-\mathrm{OH}-\mathrm{GTP}$ is not specific for the phage RNA pol. E. coli RNA pol incorporates 8-OH-GTP into E. coli DNA and 
poly(dA-dT) [13]. Calf thymus RNA pol II incorporates 8-OH-GTP into calf thymus DNA [31]. Thus, the effects of 8-OH-GTP observed in this study are also expected to be relevant for prokaryotic and eukaryotic RNA pols. Switzer et al. reported that T7 RNA pol incorporated 2-OH-ATP opposite an unnatural base, isocytosine [32]. They did not examine whether 2-OH-ATP was inserted opposite T, although they found that T7 RNA pol incorporated UTP opposite 2-OH-Ade in DNA. Since the corresponding DNA precursor, 2-hydroxy-dATP, is inserted opposite $\mathrm{T}$ and other bases in DNA templates by prokaryotic and eukaryotic DNA pols [32-34], it seems valid to speculate that 2-OH-ATP is also incorporated into RNA by prokaryotic and eukaryotic RNA pols. In addition, T7 RNA pol has been used as a model enzyme in the transcription experiments using DNA lesions, including 8-OH-Gua [28,35-38], and similar results may be obtained when prokaryotic and eukaryotic RNA pols are employed in the transcription experiments with 8-OH-GTP and 2-OH-ATP.

We used 2-OH-ATP as one of the oxidized ribonucleoside 5'-triphosphates in this study. 2-OH-ATP is produced as the major product in in vitro oxidation reaction of ATP using Fe-EDTA [15]. In addition, formation of 2-OH-Ade in cellular DNA has been reported by gas chromatography-mass spectrometry [25]. In contrast, the possibility that 2-OH-Ade is a minor oxidation product was pointed out [39]. However, 
biological significance of 2-OH-Ade nucleotides is suggested by the facts that it is formed by in in vitro oxidation reaction using Fe-nitrilotriacetate, a renal carcinogen [25] and that $\mathrm{G}: \mathrm{C} \rightarrow \mathrm{T}: \mathrm{A}$ mutations, one of the major spontaneous mutations in $\operatorname{sodAB} /$ fur E. coli cells lacking in both superoxide dismutases and repressor of iron uptake, seem to be caused by 2-hydroxy-dATP [40]. Moreover, mutant mouse MUTYH with G365D amino acid substitution, corresponding to a G382D germline mutation of human MUTYH found in familial adenomatous polyposis patients, almost completely retains its DNA glycosylase activity excising Ade opposite 8-OH-Gua and possesses $1.5 \%$ of the wild-type activity excising 2-OH-Ade opposite G [41]. Thus, 2-OH-ATP may be formed in the nucleotide pool.

Negishi and collaborators reported that ribonucleotide analogs with ambiguous base pairing properties caused mutations in reverse transcription $[42,43]$. This type of ribonucleotide analog may be a useful inducer of lethal mutagenesis or error catastrophe, and thus serve as an antiviral drug [42-45]. Since 8-OH-GTP and 2-OH-ATP caused mutations in transcription-reverse transcription, these oxidized ribonucleotides are also candidates for this type of antiviral drug.

In this study, we found that 8-OH-ATP and 2-OH-ATP suppressed the production of full-length mRNA and that these oxidized RNA 
precursors were mutagenic during in vitro transcription and reverse transcription. These results indicate that the formation of these oxidized RNA precursors in cells affects transcription quantitatively and qualitatively.

\section{Acknowledgements}

This work was supported in part by Grants-in-Aid from the Japan Society for the Promotion of Science.

Abbreviations: ROS, reactive oxygen species; 8-OH-GTP, 8-hydroxyguanosine 5'-triphosphate; pol, polymerase; 2-OH-ATP, 2-hydroxyadenosine 5'-triphosphate; RT, reverse transcriptase; 8-OH-Guo, 8-hydroxyguanosine; 8-OH-Gua, 8-hydroxyguanine; ECD, electrochemical detector; 2-OH-Ade, 2-hydroxyadenine; 8-OH-dGTP, 8-hydroxy-2'-deoxyguanosine 5'-triphosphate. 


\section{References}

1. Harman, D. The aging process. Proc. Natl. Acad. Sci. USA 78:7124-7128; 1981.

2. Ames, B.N. Dietary carcinogens and anticarcinogens. Science 221:1256-1264; 1983.

3. Kamiya, H. Mutagenic potentials of damaged nucleic acids produced by reactive oxygen/nitrogen species: Approaches using synthetic oligonucleotides and nucleotides. Nucleic Acids Res. 31:517-531; 2003.

4. McLennan, A.G. The Nudix hydrolase superfamily. Cell. Mol. Life Sci. 63:123-143; 2006.

5. Inoue, M.; Kamiya, H.; Fujikawa, K.; Ootsuyama, Y.; Murata-Kamiya, N.; Osaki, T.; Yasumoto, K.; Kasai, H. Induction of chromosomal gene mutations in Escherichia coli by direct incorporation of oxidatively damaged nucleotides. J. Biol. Chem. 273:11069-11074; 1998.

6. Fujikawa, K.; Kamiya, H.; Kasai, H. The mutations induced by oxidatively damaged nucleotides, 5-formyl-dUTP and 5-hydroxy-dCTP, in Escherichia coli. Nucleic Acids Res. 26:4582-4587; 1998. 
7. Nunomura, A.; Perry, G.; Pappollo, M. A.; Wade, R.; Hirai, K.; Chiba, S.; Smith, M. A. RNA oxidation is a prominent feature of vulnerable neurons in Alzheimer's disease. J. Neurosci. 19:1959-1964; 1999.

8. Zhang, J.; Perry, G.; Smith, M. A.; Robertson, D.; Olson, S. J.; Graham, D. G.; Montine, T. J. Parkinson's disease is associated with oxidative damage to cytoplasmic DNA and RNA in substantia nigra neurons. Am. J. Pathol. 154:1423-1429; 1999.

9. Nunomura, A. S.; Chiba, S.; Kosaka, K.; Takeda, A.; Castellani, R. J.; Smith, M. A.; Perry, G. Neuronal RNA oxidation is a prominent feature of dementia with Lewy bodies. NeuroReport 13:2035-2039; 2002.

10. Shan, X.; Tashiro, H.; Lin, C. L. The identification and characterization of oxidized RNAs in Alzheimer's disease. J. Neurosci. 23:4913-4921; 2003.

11. Mathews, C. K. Biochemistry of deoxyribonucleic acid-defective amber mutants of bacterophage T4. J. Biol. Chem. 247:7430-7438; 1972.

12. Traut, T.W. Physiological concentrations of purines and pyrimidines. Mol. Cell. Biochem. 140:1-22; 1994.

13. Taddei, F.; Hayakawa, H.; Bouton, M.; Cirinesi, A.; Matic, I.; Sekiguchi, M.; Radman, M. Counteraction by MutT protein of 
transcriptional errors caused by oxidative damage. Science 278:128-130; 1997.

14. Koyama, H.; Ito, T.; Nakanishi, T.; Kawamura, N.; Sekimizu, K. Transcription elongation factor S-II maintains transcriptional fidelity and confers oxidative stress resistance. Genes Cells 8:779-788; 2003.

15. Fujikawa, K.; Kamiya, H.; Yakushiji, H.; Nakabeppu, Y.; Kasai, H. Human MTH1 protein is highly specific for the oxidized ribonucleotide, 2-hydroxy-ATP. Nucleic Acids Res. 29:449-454; 2001.

16. Tsuchiya, H.; Harashima, H.; Kamiya, H. Increased SFHR gene correction efficiency with sense single-stranded DNA. J. Gene. Med. 7:486-493; 2005.

17. Hirano, T.; Kawai, K.; Ootsuyama, Y.; Kasai, H. Fragmentation of the DNA repair enzyme, OGG1, in mouse nonparenchymal liver cells by arsenic compounds. Genes Environ. 28:62-67; 2006.

18. Kasai, H. Analysis of a form of oxidative DNA damage, 8-hydroxy-2'-deoxyguanosine, as a marker of cellular oxidative stress during carcinogenesis. Mutat Res. 387:147-163; 1997.

19. Sen, R.; Dasgupta, D. Interaction of ribonucleotides with T7 RNA polymerase: Probable role of GTP in transcription initiation. Biochem. Biophys. Res. Commun. 195:616-622; 1993. 
20. Chen, Y.-C.; Jeng, S.-T. Binding Affinity of T7 RNA polymerase to its promoter in the supercoiled and linearized DNA templates. Biosci. Biotechnol. Biochem. 64:1126-1132; 2000.

21. Oda, Y.; Uesugi, S.; Ikehara, M.; Nishimura, S.; Kawase, Y.; Ishikawa, H.; Inoue, H.; Ohtsuka, E. NMR studies of a DNA containing 8-hydroxydeoxyguanosine. Nucleic Acids Res. 19:1407-1412; 1991.

22. Kouchakdjian, M.; Bodepudi, V.; Shibutani, S.; Eisenberg, M.; Johnson, F.; Grollman, A. P.; Patel, D. J. NMR structural studies of the ionizing radiation adduct 7-hydro-8-oxodeoxyguanosine (8-oxo-7H-dG) opposite deoxyadenosine in a DNA duplex. 8-Oxo-7H-dG(syn)•dA(anti) alignment at lesion site. Biochemistry, 30:1403-1412; 1991.

23. Plum, G. E.; Grollman, A. P.; Johnson, F.; Breslauer, K. J. Influence of the oxidatively damaged adduct 8-oxodeoxyguanosine on the conformation, energetics, and thermodynamic stability of a DNA duplex. Biochemistry 34:16148-16160; 1995.

24. Koizume, S.; Kamiya, H.; Inoue, H.; Ohtsuka, E. Synthesis and thermodynamic stabilities of damaged DNA involving 8-hydroxyguanine (7,8-dihydro-8-oxoguanine) in a ras gene fragment. Nucleosides Nucleotides 13:1517-1534; 1994. 
25. Kamiya, H.; Kasai, H. 2-Hydroxyadenine formation by reactive oxygen species and mutagenic effects. Recent Res. Dev. Biochem. 2:41-50; 2000 .

26. Kawakami, J.; Kamiya, H.; Yasuda, K.; Fujiki, H.; Kasai, H.; Sugimoto, N.; Thermodynamic stability of base pairs between 2-hydroxyadenine and incoming nucleotides as a determinant of nucleotide incorporation specificity during replication. Nucleic Acids Res. 29:3289-3296; 2001.

27. Bukowska, A. M.; Kuśmierek, J. T. Miscoding properties of isoguanine (2-oxoadenine) studied in an AMV reverse transcriptase in vitro system. Acta Biochim. Pol. 43:247-254; 1996.

28. Remington, K. M.; Bennett, S. E.; Harris, C. M. Harris and T.M., Bebenek, K. Highly mutagenic bypass synthesis by T7 RNA polymerase of site-specific benzo[a]pyrene diol epoxide-adducted template DNA. J. Biol. Chem. 273:13170-13176; 1998.

29. Huang, J.; Brieba, L. G.; Sousa, R. Misincorporation by wild-type and mutant T7 RNA polymerases: Identification of interactions that reduce misincorporation rates by stabilizing the catalytically incompetent open conformation. Biochemistry 39:11571-11580; 2000. 
30. Yu, H.; Goodman, M. F. Comparison of HIV-1 and avian myeloblastosis virus reverse transcriptase fidelity on RNA and DNA templates. J. Biol. Chem. 267:10888-10896; 1992.

31. Hayakawa, H.; Hofer, A.; Thelander, L.; Kitajima, S.; Cai, Y.; Oshiro, S.; Yakushiji, H.; Nakabeppu, Y.; Kuwano, M.; Sekiguchi, M. Metabolic fate of oxidized guanine ribonucleotides in mammalian cells. Biochemistry 38:3610-3614; 1999.

32. Switzer, C. Y.; Moroney, S. E.; Benner, S. A. Enzymic recognition of the base pair between isocytidine and isoguanosine. Biochemistry 32:10489-10496; 1993.

33. Kamiya, H.; Kasai, H. Formation of 2-hydroxydeoxyadenosine triphosphate, an oxidatively damaged nucleotide, and its incorporation by DNA polymerases. J. Biol. Chem. 270:19446-19450; 1995.

34. Kamiya, H.; Maki, H.; Kasai, H. Two DNA polymerases of Escherichia coli display distinct misinsertion specificities for 2-hydroxy-dATP during DNA synthesis. Biochemistry 39:9508-9513; 2000.

35. Chen, Y. H.; Boqenhaquen, D. F. Effects of DNA lesions on transcription elongation by T7 RNA polymerase. J. Biol. Chem. 268:5849-5855; 1993. 
36. Liu, J.; Zhou, W.; Doetsch, P. W. RNA polymerase bypass at sites of dihydrouracil: implications for transcriptional mutagenesis. Mol. Cell. Biol. 15:6729-6735; 1995.

37. Choi, D. J.; Roth, R. B.; Liu, T.; Geacintov, N. E.; Scicchitano, D. A. Incorrect Base Insertion and Prematurely Terminated Transcripts during T7 RNA Polymerase Transcription Elongation past Benzo[a]pyrenediol Epoxide-modified DNA. J. Mol. Biol. 264:213-219; 1996.

38. Tornaletti, S.; Maeda, L. S.; Kolodner, R. D.; Hanawalt, P. C. Effect of 8-oxoguanine on transcription elongation by T7 RNA polymerase and mammalian RNA polymerase II. DNA Rep. 3:483-494; 2004.

39. Frelon, S.; Douki, T.; Cadet, J. Radical oxidation of the adenine moiety of nucleoside and DNA: 2-hydroxy-2'-deoxyadenosine is a minor decomposition product. Free Radic. Res. 36:499-508; 2002.

40. Nunoshiba, T.; Watanabe, T.; Nakabeppu, Y.; Yamamoto, K. Mutagenic target for hydroxyl radicals generated in Escherichia coli mutant deficient in $\mathrm{Mn}$ - and Fe-superoxide dismutases and Fur, a repressor for iron-uptake systems. DNA Rep. 1:411-418; 2002.

41. Ushijima, Y.; Tominaga, Y.; Miura, T.; Tsuchimoto, D.; Sakumi, K.; Nakabeppu, Y. A functional analysis of the DNA glycosylase activity 
of mouse MUTYH protein excising 2-hydroxyadenine opposite guanine in DNA. Nucleic Acids Res. 33:672-682; 2005.

42. Moriyama, K.; Otsuka, C.; Loakes, D.; Negishi, K. Highly efficient random mutagenesis in transcription-reverse transcription cycles by a hydrogen bond ambivalent nucleoside 5'-triphosphate analogue: potential candidates for a selective anti-retroviral therapy. Nucleosides, Nucleotides, Nucleic Acids 20:1473-1483; 2001.

43. Suzuki, T.; Moriyama, K.; Otsuka, C.; Loakes, D.; Negishi, K. Template properties of mutagenic cytosine analogues in reverse transcription. Nucleic Acids Res. 34:6438-6449; 2006.

44. Loeb, L. A.; Essigmann, J. M.; Kazazi, F.; Zhang, J.; Rose, K. D.; Mullins, J. I. Lethal mutagenesis of HIV with mutagenic nucleoside analogs. Proc. Natl. Acad. Sci. USA 96:1492-1497; 1999.

45. Crotty, S.; Cameron, C. E.; Andino, R. RNA virus error catastrophe: direct molecular test by using ribavirin. Proc. Natl. Acad. Sci. USA 98:6895-6900; 2001. 


\section{FIGURE LEGENDS}

Figure 1 Experimental procedures in this study.

Figure 2 Proposed model for mutations induced by (A and B) 8-OH-GTP and (C) 2-OH-ATP during in vitro transcription-reverse transcription reactions. Solid and dashed lines represent DNA and RNA strands, respectively. $\mathrm{G}^{*}$ and $\mathrm{A}^{*}$ mean $8-\mathrm{OH}-\mathrm{GTP}$ and 2-OH-ATP, respectively.

Supplementary Figure S1 The overall distribution of the substitutions detected in the transcribed region. The sequence of the sense strand is shown. The mutations obtained with the control, $2 \mathrm{mM}$ 2-OH-ATP, and $2 \mathrm{mM} 8-\mathrm{OH}-\mathrm{GTP}$ experiments are shown below in black, red, and blue, respectively. The transcription initiation site is numbered as +1 . Primer sites are underlined. 
T7

promoter

$\longrightarrow$ template DNA

$\downarrow$ transcription

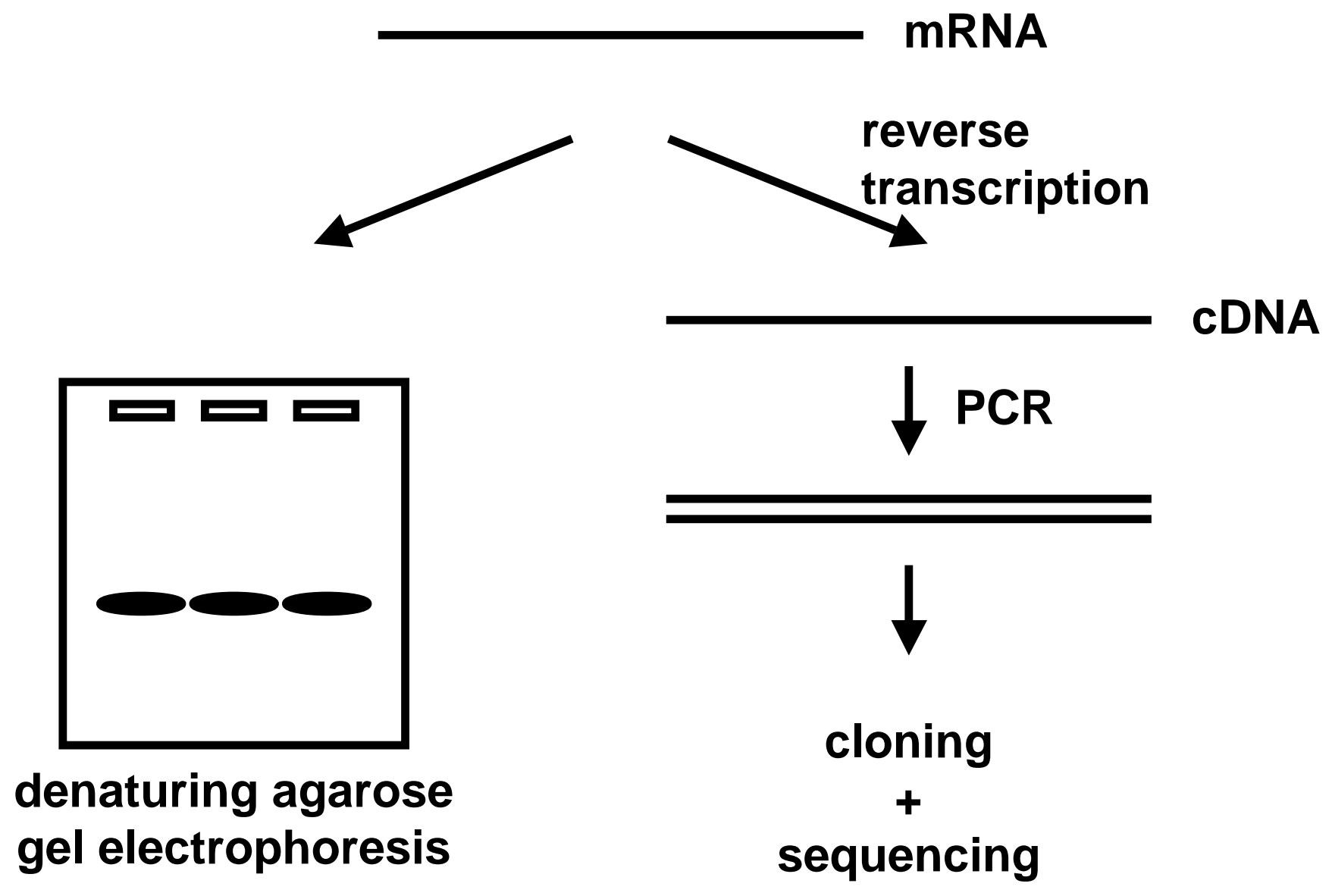

Fig. 1 
A)

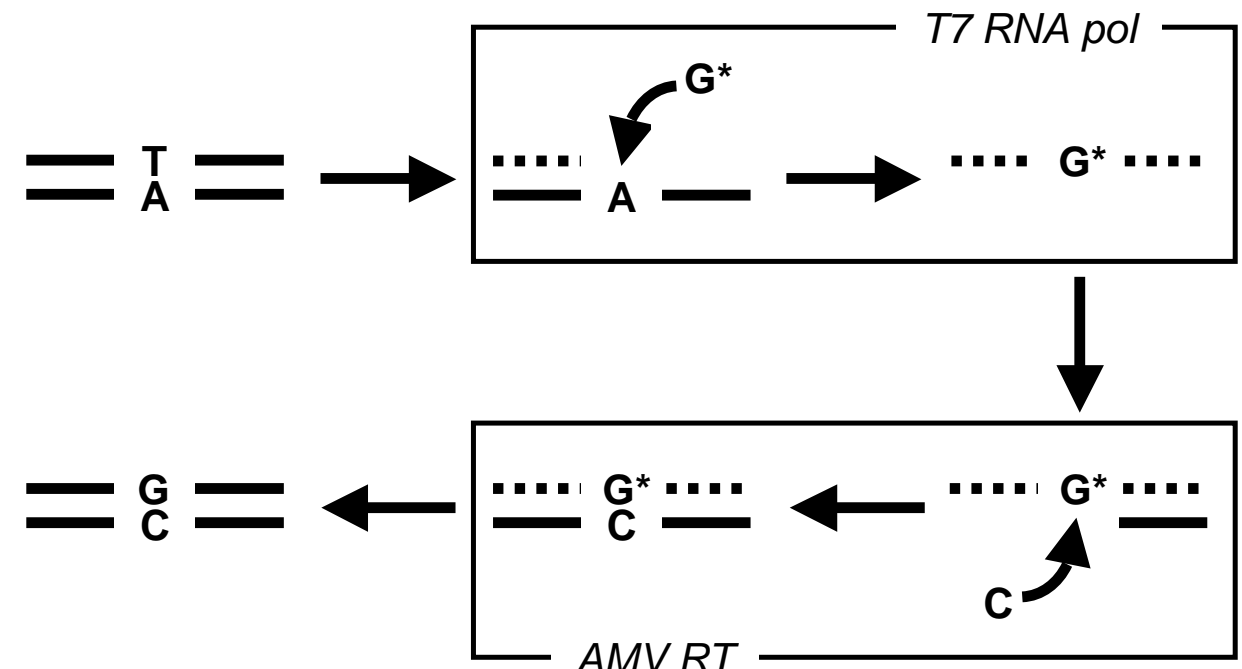

B)

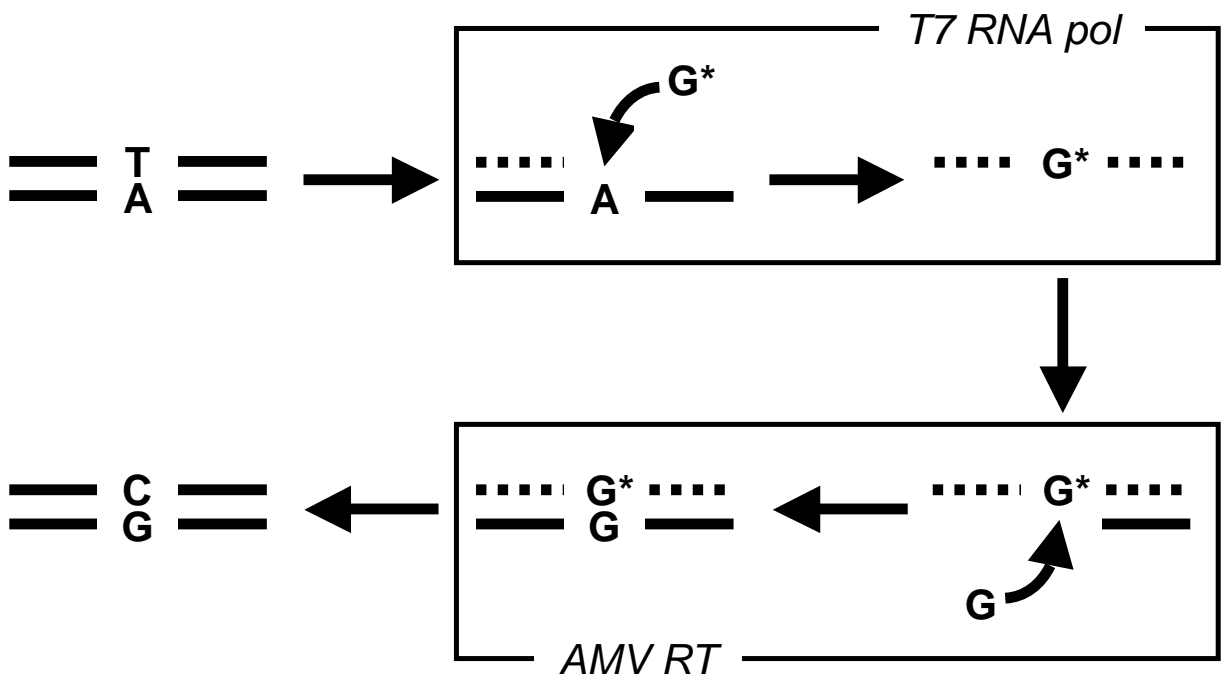

C)

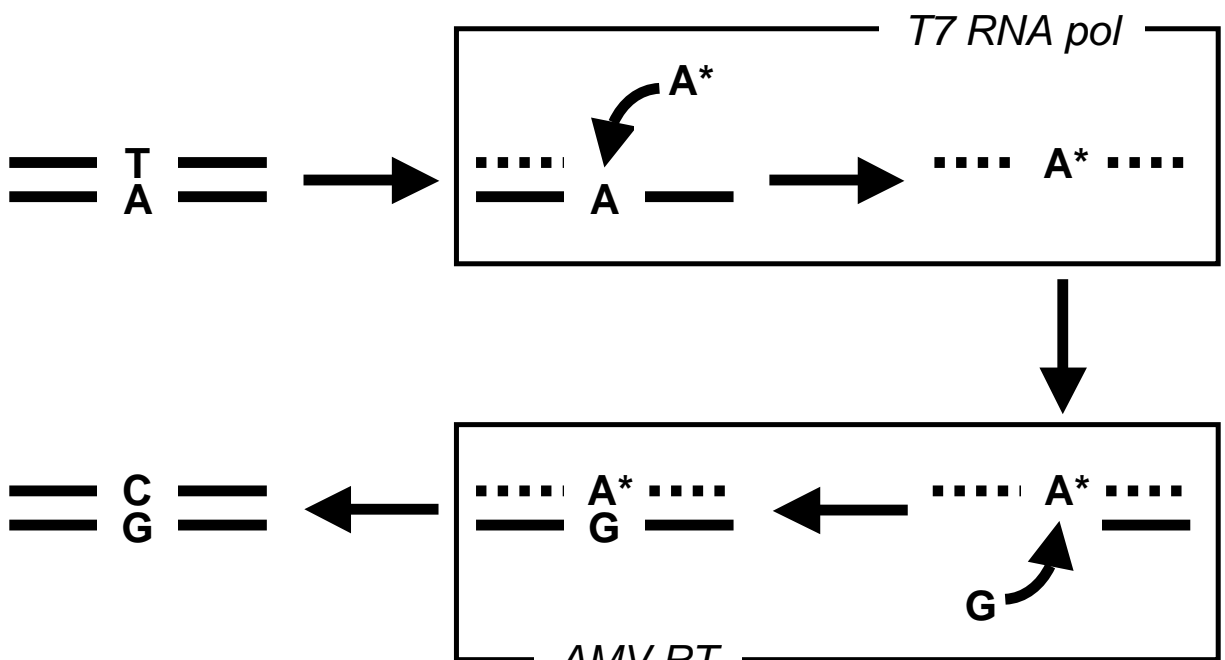

$A M V R T$

Fig. 2 
Table 1. Reduction of the amount of full-length transcript by $8-\mathrm{OH}-\mathrm{GTP}$ and $2-\mathrm{OH}-\mathrm{ATP}$ a

\begin{tabular}{cccc}
\hline \multicolumn{2}{c}{ concentrations } & & \\
\cline { 1 - 2 } Unmodified & $\begin{array}{c}\text { Oxidized } \\
\text { NTPs (mM) }\end{array}$ & 8-OH-GTP & 2-OH-ATP \\
\hline \multirow{2}{*}{2.00} & 0.00 & 100 & 100 \\
& 0.50 & 103 & 50 \\
& 1.00 & 55 & 47 \\
& 1.50 & 38 & 42 \\
& 2.00 & 16 & 25 \\
0.50 & 0.00 & 100 & 100 \\
& 0.10 & 91 & 84 \\
& 0.25 & 88 & 77 \\
& 0.50 & 43 & 58 \\
\hline
\end{tabular}

a mRNA amounts relative to those without oxidized nucleotides are shown (expressed as a percentage). The values represent the average of two $(2.00 \mathrm{mM} 2-\mathrm{OH}$ ATP) or three (others) separate experiments. 
Table 2. Mutations found after in vitro transcription and reverse transcription ${ }^{a}$

\begin{tabular}{clll}
\hline & None & 8-OH-GTP & 2-OH-ATP \\
\hline Substitution b & $3(18)$ & $19(112)^{\mathrm{c}}$ & $10(38)$ \\
$\mathrm{A} \rightarrow \mathrm{G}$ & $0(0)$ & $3(18)$ & $2(8)$ \\
$\mathrm{G} \rightarrow \mathrm{A}$ & $0(0)$ & $1(6)$ & $1(4)$ \\
$\mathrm{C} \rightarrow \mathrm{T}$ & $1(6)$ & $0(0)$ & $2(8)$ \\
$\mathrm{T} \rightarrow \mathrm{C}$ & $0(0)$ & $6(35)$ & $5(19)$ \\
$\mathrm{A} \rightarrow \mathrm{T}$ & $1(6)$ & $0(0)$ & $0(0)$ \\
$\mathrm{T} \rightarrow \mathrm{G}$ & $0(0)$ & $9(53)$ & $0(0)$ \\
$\mathrm{C} \rightarrow \mathrm{A}$ & $1(6)$ & $0(0)$ & $0(0)$ \\
None & $14(82)$ & $0(0)$ & $16(62)$ \\
Total & $17(100)$ & $17(100)$ & $26(100)$ \\
\hline
\end{tabular}

${ }^{a}$ All data are represented as cases found (\%).

$\mathrm{b}$ The sequence of the sense strand is shown.

c Two colonies contained two mutations within the analyzed regions. 
Table 3. The distribution of mutations induced by $8-\mathrm{OH}-\mathrm{GTP}$ and 2-OH-ATP a

\begin{tabular}{|c|c|c|c|}
\hline None & \multicolumn{2}{|c|}{ 8-OH-GTP } & 2-OH-ATP \\
\hline $248 \mathrm{~A} \rightarrow \mathrm{T}(1)$ & $69 \mathrm{~T} \rightarrow \mathrm{G}(2)$ & $525 \mathrm{~T} \rightarrow \mathrm{G}(1)$ & $436 \mathrm{~T} \rightarrow \mathrm{C}(1)$ \\
\hline $286 \mathrm{C} \rightarrow \mathrm{A}(1)$ & $74 \mathrm{~T} \rightarrow \mathrm{G}(1)$ & $628 \mathrm{~T} \rightarrow \mathrm{G}(1)$ & $611 \mathrm{G} \rightarrow \mathrm{A}(1)$ \\
\hline \multirow[t]{7}{*}{$861 \mathrm{C} \rightarrow \mathrm{T}(1)$} & $158 A \rightarrow G(1)$ & $651 \mathrm{~T} \rightarrow \mathrm{C}(1)$ & $711 \mathrm{~T} \rightarrow \mathrm{C}(1)$ \\
\hline & $174 \mathrm{~T} \rightarrow \mathrm{C}(1)$ & $711 \mathrm{~T} \rightarrow \mathrm{G}(1)$ & $715 \mathrm{~T} \rightarrow \mathrm{C}(2)$ \\
\hline & $269 \mathrm{~T} \rightarrow \mathrm{C}(1)$ & $713 A \rightarrow G(1)$ & $738 A \rightarrow G(2)$ \\
\hline & $276 \mathrm{~T} \rightarrow \mathrm{G}(1)$ & $737 \mathrm{C} \rightarrow \mathrm{A}(1)$ & $927 \mathrm{~T} \rightarrow \mathrm{C}(1)$ \\
\hline & $311 \mathrm{~T} \rightarrow \mathrm{C}(1)$ & $987 \mathrm{~T} \rightarrow \mathrm{C}(1)$ & $1045 \mathrm{C} \rightarrow \mathrm{T}(2)$ \\
\hline & $374 \mathrm{~T} \rightarrow \mathrm{G}(1)$ & $1035 \mathrm{~T} \rightarrow \mathrm{G}(1)$ & \\
\hline & $392 \mathrm{~T} \rightarrow \mathrm{C}(1)$ & $1071 A \rightarrow G(1)$ & \\
\hline
\end{tabular}

a The sequence of the sense strand is shown. The numbers of colonies are shown on the right side in parentheses. 


\section{None ... black}

2-OH-ATP ... red

8-OH-GTP ... blue

GgGgAATtgT GAgCGgAtAA CAATtCCCCG GAgtTAATCC GgGACCTtTA

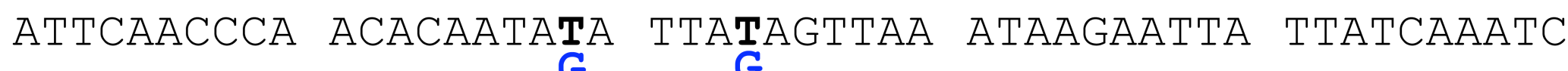
G

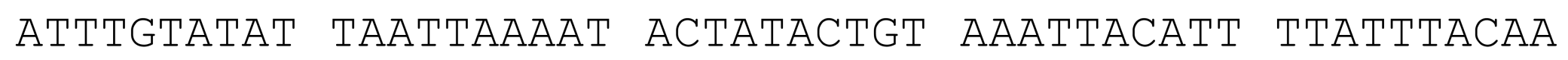

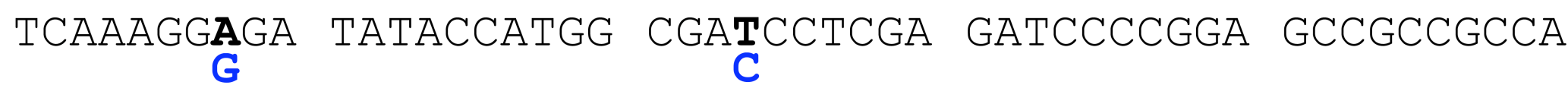

CCATGAAAAA GCCTGAACtC ACCGCGACGT CtgtCGAgAA GTtTCtgAtC

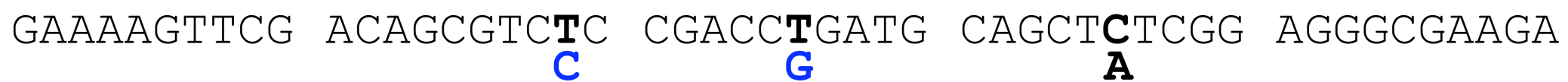

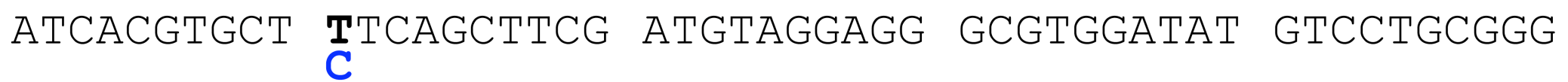

Fig. $\$ 1(1 / 3)$ 


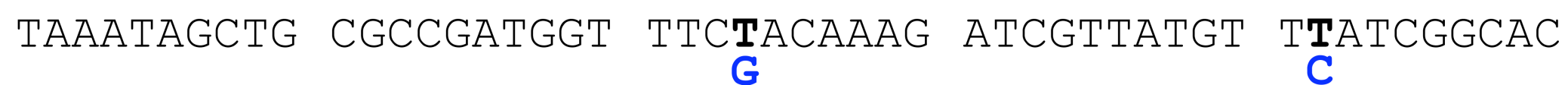

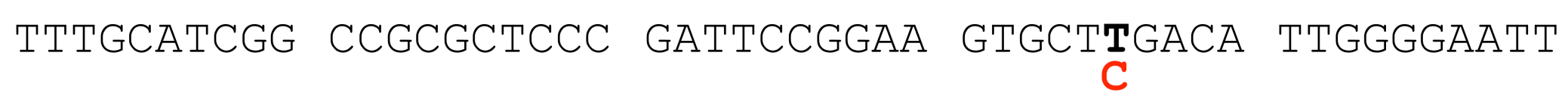

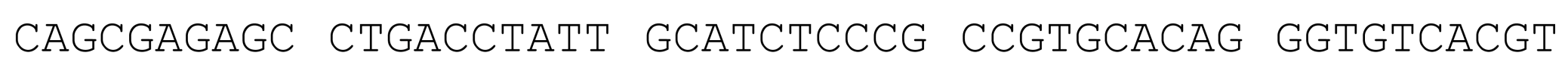

TGCAAGACCT GCCTGAAACC GAACT,GCCCG CTGTTCTGCA GCCGGTCGCG

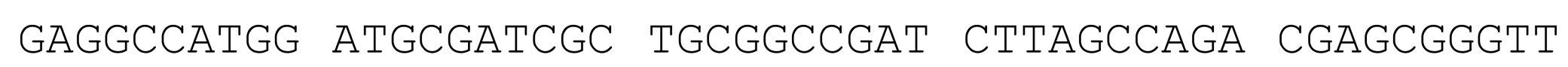

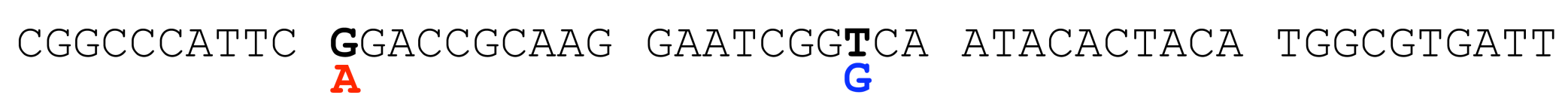

TCATATGCGC GATTGCTGAT CCCCATGTGT ATCACTGGCA AACTGTGATG

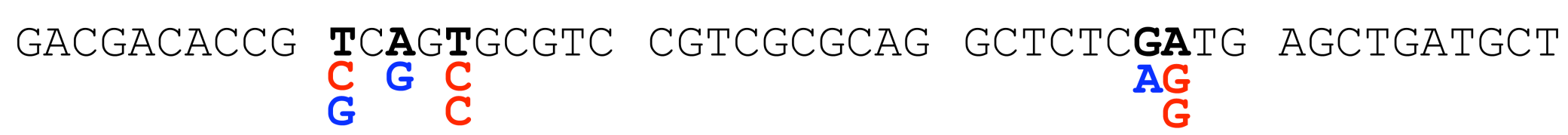

TtgGgCCGA GACTGCCCCG AAgtCCGgCA CCtCGAgCAC GCGgAtTtCG 
GCTCCAACAA TGTCCTGACG GACAATGgCC GCATAACAGC GgtCATtGAC

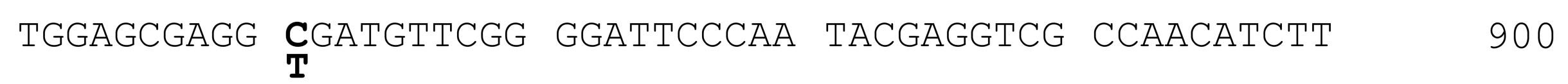

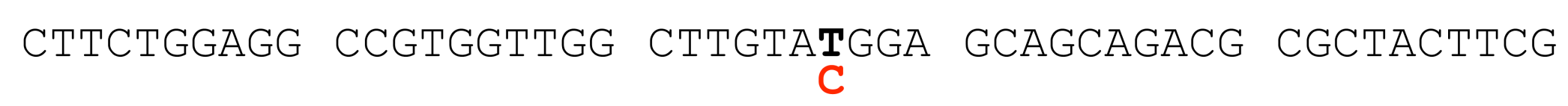

AgCGgAgGCA TCCGGAGCTT GCAGGATCGC CGCGGCTCCCG GGCGTATATG 1000

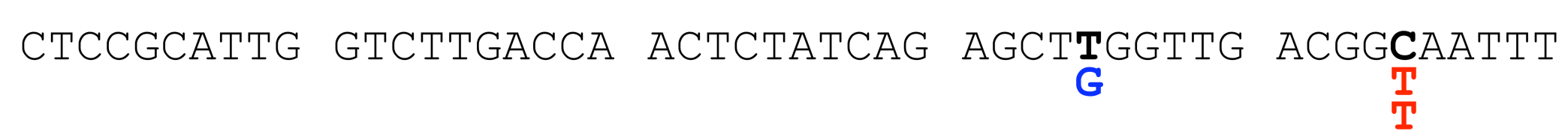

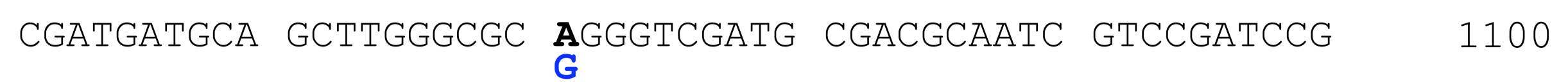

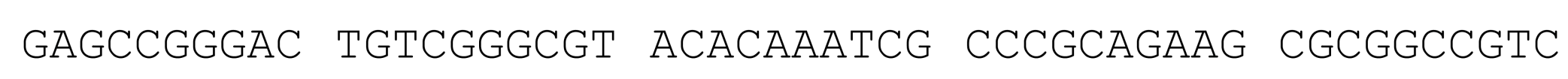

TGGACCGATG GCTGTGTAGA AGT

Fig. $\mathrm{S} 1(3 / 3)$ 